\title{
Ultraviolet completion of a composite asymmetric dark matter model with a dark photon portal
}

\author{
Masahiro Ibe, ${ }^{a, b}$ Ayuki Kamada, ${ }^{c}$ Shin Kobayashi, ${ }^{a, b}$ Takumi Kuwahara ${ }^{c}$ and \\ Wakutaka Nakano ${ }^{a, b}$ \\ ${ }^{a}$ Kavli IPMU (WPI), UTIAS, The University of Tokyo, \\ Kashiwa, Chiba 27\%-8583, Japan \\ ${ }^{b}$ ICRR, The University of Tokyo, \\ Kashiwa, Chiba 277-8582, Japan \\ ${ }^{c}$ Center for Theoretical Physics of the Universe, Institute for Basic Science (IBS), \\ 55 Expo-ro, Yuseong-gu, Daejeon 34126, Korea \\ E-mail: ibe@icrr.u-tokyo.ac.jp, akamada@ibs.re.kr, \\ shinkoba@icrr.u-tokyo.ac.jp, kuwahara@ibs.re.kr, \\ m156077@icrr.u-tokyo.ac.jp
}

ABSTRACT: Composite asymmetric dark matter scenarios naturally explain why the dark matter mass density is comparable with the visible matter mass density. Such scenarios generically require some entropy transfer mechanism below the composite scale; otherwise, their late-time cosmology is incompatible with observations. A tiny kinetic mixing between a dark photon and the visible photon is a promising example of the low-energy portal. In this paper, we demonstrate that grand unifications in the dark and the visible sectors explain the origin of the tiny kinetic mixing. We particularly consider an ultraviolet completion of a simple composite asymmetric dark matter model, where asymmetric dark matter carries a $B-L$ charge. In this setup, the longevity of asymmetric dark matter is explained by the $B-L$ symmetry, while the dark matter asymmetry originates from the $B-L$ asymmetry generated by thermal leptogenesis. In our minimal setup, the Standard Model sector and the dark sector are unified into $\mathrm{SU}(5)_{\mathrm{GUT}} \times \mathrm{SU}(4)_{\text {DGUT }}$ gauge theories, respectively. This model generates required $B-L$ portal operators while suppressing unwanted higher-dimensional operators that could wash out the generated $B-L$ asymmetry.

KeYwords: Beyond Standard Model, GUT

ARXIV EPRINT: 1811.10232 


\section{Contents}

1 Introduction 1

2 Simple composite ADM model 3

3 Non-supersymmetric realization $\quad 5$

3.1 Tiny kinetic mixing $\quad 6$

$3.2 B-L$ portal operator $\quad 6$

3.3 Dark matter phenomenology 8

$\begin{array}{llr}3.4 \text { Remarks } & 10\end{array}$

4 Supersymmetric realization $\quad 11$

4.1 Tiny kinetic mixing and $B-L$ portal operator $\quad 12$

$\begin{array}{lll}4.2 & \text { Lightest supersymmetric particles in two sectros } & 12\end{array}$

5 Conclusion $\quad 14$

\section{Introduction}

While astrophysical and cosmological observations have firmly established the existence of dark matter (DM), only a few properties of DM particles have been revealed: they should be stable or have a lifetime longer than the age of the Universe; and they interact with the standard model (SM) particles only weakly.

Stability is one of the critical ingredients to identify the nature of DM particles. It is well known that the proton has a long lifetime, and its longevity is ensured by an accidental symmetry, called the baryon number symmetry, in the SM. It is natural to consider that DM particles are stable for a similar reason: they are dark baryons in a strong dynamics in the dark sector, and the dark baryon number is conserved accidentally [1-39] (see ref. [40] for a review).

Composite DM is particularly well motivated in the asymmetric dark matter (ADM) framework, where the asymmetry generated in the visible and/or the dark sectors is communicated to the other sector via some portal interactions (see refs. [41-52] for early works and also refs. [53-55] for reviews). The common origin of the DM and the baryon asymmetries explains why the DM mass density today is about five times larger than the visible matter mass density when the DM mass is in the GeV range. Dimensional transmutation of the strong dynamics can in turn naturally explain the DM mass at the GeV scale. Besides, the dark baryons annihilate into dark pions quite efficiently, so that the asymmetric component dominates the DM relic density. The dark baryon self-interaction mediated by dark mesons may also realize the velocity dependent cross section that addresses the dwarf 
galaxy-scale issues of structure formation of collisionless cold dark matter while leaves its success at galaxy clusters (see ref. [56] for a review).

In composite ADM scenarios, the dark sector is in thermal equilibrium with the visible sector through high-energy portal interactions that communicate the asymmetry. Accordingly, the dark sector possesses a sizable entropy density comparable to the one in the visible sector. As the entropy densities are conserved separately in the two sectors after the decoupling of the high-energy portal interaction, the resultant dark sector entropy density is carried over by the light particles in the dark sector such as dark pions. The latetime energy density of the light particles could overclose the Universe or give a significant contribution to the dark radiation, depending on their masses [57].

To overcome such a shortcoming of composite ADM, one needs to introduce an additional low-energy portal that transfers the dark sector entropy density into the visible sector. One promising candidate is a dark photon portal, where the dark photon decays into a pair of the electron and the positron through the kinetic mixing with the visible photon [58] (see also refs. $[36,59,60]$ in the context of composite ADM). It is shown that the $\mathrm{MeV}$-scale dark photon with the kinetic mixing of the order of $10^{-9}$ is a viable portal evading all the experimental and cosmological constraints [36].

On the other hand, the origin of the tiny kinetic mixing is unclear. In this paper, we propose grand unifications (GUTs) in the two sectors as its origin. If the SM U $(1)_{Y}$ and the dark $\mathrm{U}(1)_{D}$ dynamics are unified into separate non-abelian gauge dynamics at some high energies, the kinetic mixing vanishes above the unification scales. It arises through a higher dimensional non-renormalizable operator and is suppressed by the ratios between the GUT scales and the Planck scale. For example, the dark GUT scale of the order of $10^{10} \mathrm{GeV}$ provides a kinetic mixing of the order of $10^{-9}$ when the unified gauge groups are broken by the vacuum expectation values (VEVs) of adjoint scalars.

This dark GUT scale is comparable with the minimal right-handed neutrino mass required for thermal leptogenesis, $M_{R}>10^{9} \mathrm{GeV}$ [61] (see refs. [62-64] for reviews). We obtain the high-energy portal interactions that communicate the $B-L$ asymmetry by integrating out the dark GUT particles and right-handed neutrinos in our setup. Thus one can take advantage of right-handed neutrinos as the origins of the $B-L$ asymmetry and the high-energy portal operator. If the dark GUT scale is much higher than the right-handed neutrino mass scale, for example, the generated $B-L$ portal operators are too much suppressed to equilibrate the $B-L$ asymmetry between the two sectors. As a particular example, we take a minimal composite ADM model proposed in ref. [36]. The model is based on the dark quantum chromodynamics (dark QCD) and the dark quantum electrodynamics (dark QED). An ADM candidate in the model is dark nucleons that consist of dark quarks carrying $B-L$ number. In this paper, we provide an ultraviolet (UV) completion of the simple ADM model proposed in ref. [36] based on a product GUT of $\mathrm{SU}(5)_{\mathrm{GUT}} \times \mathrm{SU}(4)_{\mathrm{DGUT}}$.

Furthermore, the UV completion clarifies the origin of the high-energy portal operators with which the visible and the dark sectors share the $B-L$ asymmetries. The portal interactions in composite ADM models are generically provided as non-renormalizable operators. From the view point of the effective field theory, there is no reason why some of 


\begin{tabular}{|c|c|c|c|}
\hline & $\mathrm{SU}(3)_{D}$ & $\mathrm{U}(1)_{D}$ & $\mathrm{U}(1)_{B-L}$ \\
\hline$U^{\prime}$ & $\mathbf{3}$ & $2 / 3$ & $1 / 3$ \\
$\bar{U}^{\prime}$ & $\overline{\mathbf{3}}$ & $-2 / 3$ & $-1 / 3$ \\
$D^{\prime}$ & $\mathbf{3}$ & $-1 / 3$ & $1 / 3$ \\
$\bar{D}^{\prime}$ & $\overline{\mathbf{3}}$ & $1 / 3$ & $-1 / 3$ \\
\hline
\end{tabular}

Table 1. Charge assignment of the dark quarks for a composite ADM model. $\mathrm{SU}(3)_{D}$ and $\mathrm{U}(1)_{D}$ are gauge symmetries of the dark sector, while $\mathrm{U}(1)_{B-L}$ is the global symmetry shared with the visible sector.

unwanted non-renormalizable operators which could wash out the $B-L$ asymmetry are much suppressed than the required portal operators. One can address this issue only by specifying the UV completions of the model of ADM. Thus, it is important to construct viable UV completions of the composite ADM models in order to guarantee that such dangerous operators are safely neglected.

This paper is organized as follows. In section 2, we briefly review a composite model of ADM [36]. We construct UV models of a composite ADM model: a non-supersymmetric (non-SUSY) model in section 3 and a SUSY model in section 4 . Section 5 is devoted to conclusions of our work.

\section{Simple composite ADM model}

In this section, we sketch out a composite ADM model and fix our notation. We consider the vector-like two-flavor $\mathrm{SU}(3)_{D} \times \mathrm{U}(1)_{D}$ dynamics proposed in ref. [36]. The dark quarks consist of (anti-)fundamental representations in $\mathrm{SU}(3)_{D}$, which have the same $B-L$ numbers as the up-type and the down-type quarks in the visible QCD. We list the minimal particle contents in the dark sector for the model in table 1 . The chiral symmetry of the dark quarks are softly broken by the current quark masses,

$$
\mathcal{L}_{\text {mass }}=m_{U^{\prime}} \bar{U}^{\prime} U^{\prime}+m_{D^{\prime}} \bar{D}^{\prime} D^{\prime}+\text { h.c. }
$$

The dark quarks are confined into dark mesons and dark baryons below the dynamical scale of $\mathrm{SU}(3)_{D}$, i.e., $\Lambda_{\mathrm{DQCD}}$. Dark baryons carry $B-L$ numbers, and thus the lightest one is stable in the dark sector and is a good candidate for DM.

The lightest dark mesons are also stable in composite models, and hence, they could lead to the overclosure of the Universe or a too large effective number of neutrino species $N_{\text {eff. }}$ The dark QED is introduced in order to avoid the cosmological problems. When the dark quarks are charged under $\mathrm{U}(1)_{D}$, the dark mesons annihilate into the dark photons. The $\mathrm{U}(1)_{D}$ charges for the dark quarks are determined by the required existence of a neutral dark baryon, which is essential for the high-energy portal operator described below.

The dark photon can decay into the visible particles when the kinetic mixing with the visible photon and a mass of the dark photon are introduced,

$$
\mathcal{L}_{\gamma^{\prime}}=\frac{\epsilon}{2} F_{\mu \nu} F^{\prime \mu \nu}+\frac{m_{\gamma^{\prime}}^{2}}{2} A_{\mu}^{\prime} A^{\prime \mu}
$$


Here, $F_{\mu \nu}$ and $F_{\mu \nu}^{\prime}$ are the field strengths of the SM photon $A_{\mu}$ and the dark photon $A_{\mu}^{\prime}$, respectively. The dark photon parameters $\left(\epsilon, m_{\gamma^{\prime}}\right)$ are severely constrained by beam dump experiments, collider experiments [65], SN 1987A [66, 67], and the effects on the effective number of neutrino species $N_{\text {eff }}$ [36]. In this work we assume the viable dark photon parameters in the ranges of $\epsilon=10^{-10_{-}}-10^{-9}$ and $m_{\gamma^{\prime}}=\mathcal{O}\left(10^{2}-10^{3}\right) \mathrm{MeV}$ [36]. The origin of the tiny kinetic mixing is unclear, while is naturally understood in a GUT model investigated in the next section.

In the model proposed in ref. [36], the $B-L$ asymmetry in the visible sector is assumed to be generated by thermal leptogenesis. The right-handed neutrinos couple to the SM lepton doublet $L$ and the SM Higgs doublet $H$ as

$$
\mathcal{L}_{N}=\frac{M_{R}}{2} \overline{N N}+y_{N} L H \bar{N}+\text { h.c. }
$$

with Majorana masses being $M_{R} \gtrsim 10^{9} \mathrm{GeV}$. We introduce at least two generations of the right-handed neutrinos to make thermal leptogenesis work. Hereafter we suppress the generation indices for the sake of notational simplicity. Remark that the right-handed neutrinos can also generate tiny neutrino masses through the see-saw mechanism [68-71]. It relates the Yukawa coupling with the observed neutrino mass $m_{\nu}$ as

$$
y_{N}^{2} \sim 10^{-5}\left(\frac{m_{\nu}}{0.1 \mathrm{eV}}\right)\left(\frac{M_{R}}{10^{9} \mathrm{GeV}}\right) .
$$

The generated $B-L$ asymmetry is shared between the dark and the visible sectors through portal operators,

$$
\mathcal{L}_{\text {portal }}=\frac{c_{1} y_{N}}{\Lambda^{2} M_{R}}\left(\bar{U}^{\prime} \bar{D}^{\prime} \bar{D}^{\prime}\right)(L H)+\frac{c_{2} y_{N}}{\Lambda^{2} M_{R}}\left(U^{\prime \dagger} D^{\prime \dagger} \bar{D}^{\prime}\right)(L H)+\text { h.c. },
$$

which are obtained from

$$
\mathcal{L}_{\text {portal }}=\frac{c_{1}}{\Lambda^{2}}\left(\bar{U}^{\prime} \bar{D}^{\prime} \bar{D}^{\prime}\right) \bar{N}+\frac{c_{2}}{\Lambda^{2}}\left(U^{\prime \dagger} D^{\prime \dagger} \bar{D}^{\prime}\right) \bar{N}+\text { h.c. },
$$

below the energy scale of $M_{R}$. Here $\Lambda$ is the portal scale and $c_{1}$ and $c_{2}$ are $\mathcal{O}(1)$ dimensionless constants.

After a part of $B-L$ is stored in the dark sector, the dark nucleons form below the dark confinement scale, and then the lightest one is the ADM candidate. The lightest one is almost stable since the $B-L$ number is approximately conserved in the dark sector and the portal interactions to visible matters are suppressed by $\Lambda$. The dark neutron, which consists of $U^{\prime} D^{\prime} D^{\prime}$ and $\bar{U}^{\prime \dagger} \bar{D}^{\prime \dagger} \bar{D}^{\prime \dagger}$, and the dark proton, which consists of $U^{\prime} U^{\prime} D^{\prime}$ and $\bar{U}^{\prime \dagger} \bar{U}^{\prime \dagger} \bar{D}^{\prime \dagger}$ are ADM candidates in this setup. The mass of ADM particles is determined by the ratio of the asymmetries in the $\mathrm{SM}$ and the dark sectors: $m_{\mathrm{DM}}=8.5 \mathrm{GeV}[36,72,73]$. It implies that the dark dynamical scale $\Lambda_{\mathrm{DQCD}}$ is an order of magnitude larger than the QCD scale, namely $\Lambda_{\mathrm{DQCD}} \sim 2 \mathrm{GeV}$.

The portal scale $\Lambda$ is bounded from below by neutrino flux measurements [73]. Meanwhile the portal interactions should decouple after the $B-L$ asymmetry is generated, namely, the decoupling temperature should be below $M_{R}$. The decoupling temperature is 
estimated as $T_{*} \sim M_{*}\left(M_{*} / M_{\mathrm{Pl}}\right)^{1 / 5}$ with $M_{*}$ collectively denoting $\left(\Lambda^{2} M_{R} / c_{i} y_{N}\right)^{1 / 3}(i=1,2)$ and $M_{\mathrm{Pl}}=2.4 \times 10^{18} \mathrm{GeV}$ being the reduced Planck mass. Noting eq. (2.4) and requiring $M_{R}<\Lambda$ for the consistency of the renormalizable operator, one obtains

$$
M_{R}<\Lambda \lesssim 10 c_{i}^{1 / 2} M_{R}, \quad \text { and thus } \quad c_{i} \gtrsim 0.01
$$

The origin of the portal operators can be easily explained if a scalar field charged under $\mathrm{SU}(3)_{D}$ with a mass about $\Lambda$ is introduced. In the next section we will construct a $\mathrm{SU}(4)_{\text {DGUT }}$ unified model of $\mathrm{SU}(3)_{D}$ and $\mathrm{U}(1)_{D}$ gauge dynamics in the dark sector.

As the $B-L$ symmetry is softly broken by the right-handed neutrino masses, no symmetry prohibits the operators that carry different $B-L$ charges so far. If the following operators are also relevant after the $B-L$ asymmetry is generated, the asymmetry is washed out:

$$
\mathcal{L}=\frac{c_{1}^{\prime}}{\Lambda^{\prime 2}}\left(U^{\prime} D^{\prime} D^{\prime}\right) \bar{N}+\frac{c_{2}^{\prime}}{\Lambda^{\prime 2}}\left(\bar{U}^{\prime \dagger} \bar{D}^{\prime \dagger} D^{\prime}\right) \bar{N}+\text { h.c. }
$$

Here $c_{1}^{\prime}$ and $c_{2}^{\prime}$ are $\mathcal{O}(1)$ dimensionless constants. We approximately obtain the cutoff scale $\Lambda^{\prime}$ should be larger than $10^{11} \mathrm{GeV}$ by requiring that the decoupling temperature of the interactions should be higher than $M_{R} \sim 10^{9} \mathrm{GeV}$. The hierarchy between $\Lambda$ and $\Lambda^{\prime}$, namely, between the desirable operators and dangerous operators for an ADM scenario, will also be understood in a natural way by a UV completion in the dark sector and its symmetry. ${ }^{1}$

\section{Non-supersymmetric realization}

We consider a non-SUSY SU(5) $)_{\text {GUT }} \times \mathrm{SU}(4)_{\mathrm{DGUT}}$ GUT model. Here, SU(5) GUT $_{\text {stands }}$ for the grand unified gauge group of the SM sector in the Georgi-Glashow model [74]. The $\mathrm{SU}(5)_{\text {GUT }}$ gauge symmetry is spontaneously broken down to the SM gauge group $\mathrm{SU}(3) \times$ $\mathrm{SU}(2) \times \mathrm{U}(1)$ by the VEV of an adjoint scalar field $\Sigma(\mathbf{2 4})$, i.e., $\langle\Sigma\rangle=v_{24} \operatorname{diag}(2,2,2,-3,-3)$. We note that the GUT scale $v_{24}$ in the visible sector is assumed to be of the order of $10^{16} \mathrm{GeV}$ to avoid too rapid proton decay [75] even though intermediate-scale SUSY is not introduced. Such a large GUT scale is achieved if some additional fields are introduced at an intermediate scale (see refs. [76-82]).

We take the minimal option for the dark sector, $\mathrm{SU}(4)_{\text {DGUT }}$, which includes $\mathrm{SU}(3)_{D} \times$ $\mathrm{U}(1)_{D}$ as a subgroup. The $\mathrm{SU}(4)_{\text {DGUT }}$ symmetry is broken by an adjoint scalar field $\Xi^{\prime}(\mathbf{1 5})$ by its VEV, i.e., $\left\langle\Xi^{\prime}\right\rangle=v_{15} \operatorname{diag}(1,1,1,-3)$. We assume that $v_{15}$ is of the order of $10^{10} \mathrm{GeV}$ and is much smaller than $v_{24}$. We note that the dark sector is an asymptotically free theory, and hence, the perturbativity in the dark sector is ensured up to the Planck scale.

\footnotetext{
${ }^{1}$ When $\bar{N}$ 's are integrated out, the dark neutrons $n^{\prime} \propto \bar{U}^{\prime} \bar{D}^{\prime} \bar{D}^{\prime}$ obtains a tiny $B-L$ breaking mass of $\mathcal{O}\left(\Lambda_{\mathrm{DQCD}}^{6} / M_{R} \Lambda^{5}\right)$. The $B-L$ breaking mass is, however, small enough not to wash out the $B-L$ asymmetry.
} 


\subsection{Tiny kinetic mixing}

The smallness of the visible photon-dark photon kinetic mixing $\epsilon$ is naturally explained in this setup. We assume that any non-renormalizable operator is suppressed by the reduced Planck mass $M_{\mathrm{Pl}}$ above the $\mathrm{SU}(5)_{\mathrm{GUT}} \times \mathrm{SU}(4)_{\text {DGUT }}$ GUT scale. Under this assumption, the kinetic mixing arises from the following operator:

$$
\mathcal{L}_{\epsilon}=\frac{1}{M_{\mathrm{Pl}}^{2}} \operatorname{tr}\left(F_{G \mu \nu} \Sigma\right) \operatorname{Tr}\left(F_{D}^{\mu \nu} \Xi^{\prime}\right),
$$

where $\operatorname{tr}$ and $\mathrm{Tr}$ denote traces of $\mathrm{SU}(5)_{\text {GUT }}$ and $\mathrm{SU}(4)_{\text {DGUT }}$ indices, respectively. $F_{G \mu \nu}$ and $F_{D \mu \nu}$ are the field strengths of $\mathrm{SU}(5)_{\mathrm{GUT}}$ and $\mathrm{SU}(4)_{\mathrm{DGUT}}$, respectively. Below the GUT scale, the kinetic mixing is given by ${ }^{2}$

$$
\epsilon=\frac{2 \sqrt{10} v_{24} v_{15} \cos \theta_{W}}{M_{\mathrm{Pl}}^{2}} \simeq 10^{-9}\left(\frac{v_{24}}{2 \times 10^{16} \mathrm{GeV}}\right)\left(\frac{v_{15}}{5 \times 10^{10} \mathrm{GeV}}\right),
$$

with the Weinberg angle being $\sin ^{2} \theta_{W} \simeq 0.23$. We naturally obtain the tiny mixing parameter $\epsilon$ thanks to the hierarchy of $v_{15} \ll v_{24}<M_{\mathrm{Pl}}$.

\section{$3.2 \quad B-L$ portal operator}

In the GUT picture, $\mathrm{U}(1)_{B-L}$ is realized as the "fiveness" $\mathrm{U}(1)_{5}$ that commutes with the $\mathrm{SU}(5)_{\mathrm{GUT}}$ and the $\mathrm{SU}(4)_{\mathrm{DGUT}}$ symmetries. The fiveness charge $Q_{5}$ is related to the hypercharge $Y$ and the $\mathrm{U}(1)_{D}$ charge $D$ via $Q_{5}=5(B-L)-4 Y-\frac{5}{2} D$. Table 2 shows the minimal particle contents of SU(4) DGUT and their charge assignment.

The dark quarks listed in table 1 are unified into the $\mathbf{6}, \mathbf{4}$, and $\overline{\mathbf{4}}$ representations of $\mathrm{SU}(4)_{\mathrm{DGUT}}$ that are denoted by $Q_{U}, Q_{D}$, and $\bar{Q}_{\bar{D}}$, respectively. Indeed, under the symmetry breaking of $\mathrm{SU}(4)_{\mathrm{DGUT}} \rightarrow \mathrm{SU}(3)_{D} \times \mathrm{U}(1)_{D}$, a fundamental representation in $\mathrm{SU}(4)_{\text {DGUT }}$ is decomposed as $\mathbf{4} \rightarrow \mathbf{3}_{-1 / 3}+\mathbf{1}_{1}$, while an anti-symmetric representation in $\mathrm{SU}(4)_{\text {DGUT }}$ is decomposed as $\mathbf{6} \rightarrow \mathbf{3}_{2 / 3}+\overline{\mathbf{3}}_{-2 / 3}$ :

$$
Q_{U}^{\prime}=\frac{1}{\sqrt{2}}\left(\begin{array}{cccc}
0 & \bar{U}^{\prime 3} & -\bar{U}^{\prime 2} & U_{1}^{\prime} \\
-\bar{U}^{\prime 3} & 0 & \bar{U}^{\prime 1} & U_{2}^{\prime} \\
\bar{U}^{\prime 2} & -\bar{U}^{\prime 1} & 0 & U_{3}^{\prime} \\
-U_{1}^{\prime} & -U_{3}^{\prime} & -U_{3}^{\prime} & 0
\end{array}\right), \quad Q_{D}^{\prime}=\left(\begin{array}{c}
D_{1}^{\prime} \\
D_{2}^{\prime} \\
D_{3}^{\prime} \\
\bar{E}^{\prime}
\end{array}\right), \quad \bar{Q}_{\bar{D}}^{\prime}=\left(\begin{array}{c}
\bar{D}^{\prime 1} \\
\bar{D}^{\prime 2} \\
\bar{D}^{\prime 3} \\
E^{\prime}
\end{array}\right)
$$

Here, $E^{\prime}\left(\bar{E}^{\prime}\right)$ is a $\mathrm{SU}(3)_{D}$ singlet with $\mathrm{U}(1)_{D}$ charge $-1(+1)$, and thus we refer to them as the dark electron. The sub- and super-scripts denote $\mathrm{SU}(3)_{D}$ indices.

We also introduce a fundamental scalar field $H^{\prime}$, which is decomposed into a darkcolored Higgs triplet $\phi_{C}$ and a dark $\mathrm{U}(1)_{D}$ breaking Higgs $\phi_{D} \cdot{ }^{3}$ We impose fine-tuning of parameters in order to realize the mass difference between $\phi_{C}$ and $\phi_{D}$, namely between $10^{10} \mathrm{GeV}$ and $1 \mathrm{GeV}$.

\footnotetext{
${ }^{2}$ Radiative contributions also arise via three-loop diagrams involving right-handed neutrinos. They are suppressed by loop factors and small $y_{N}$ [see eq. (2.4)], and thus are subdominant.

${ }^{3}$ It is possible to introduce another representation for the dark $\mathrm{U}(1)_{D}$ breaking Higgs and the darkcolored Higgs triplet. For instance, a symmetric representation is decomposed as $\mathbf{1 0} \rightarrow \mathbf{6}_{-2 / 3}+\mathbf{3}_{2 / 3}+\mathbf{1}_{2}$. On the other hand, the $B-L$ portal interactions do not arise from the dark colored Higgs triplet from the 10 representation without introducing extra fermions.
} 


\begin{tabular}{|c|c|c|c|}
\hline & $\mathrm{SU}(5)_{\text {GUT }}$ & $\mathrm{SU}(4)_{\text {DGUT }}$ & $\mathrm{U}(1)_{5}$ \\
\hline$\Psi_{i}$ & $\mathbf{1 0}$ & $\mathbf{1}$ & 1 \\
$\Phi_{i}$ & $\overline{\mathbf{5}}$ & $\mathbf{1}$ & -3 \\
$\bar{N}_{i}$ & $\mathbf{1}$ & $\mathbf{1}$ & 5 \\
\hline$Q_{U}^{\prime}$ & $\mathbf{1}$ & $\mathbf{6}$ & 0 \\
$Q_{D}^{\prime}$ & $\mathbf{1}$ & $\mathbf{4}$ & $5 / 2$ \\
$\bar{Q}_{\bar{D}}^{\prime}$ & $\mathbf{1}$ & $\overline{\mathbf{4}}$ & $-5 / 2$ \\
\hline
\end{tabular}

\begin{tabular}{|c|c|c|c|}
\hline & $\mathrm{SU}(5)_{\text {GUT }}$ & $\mathrm{SU}(4)_{\text {DGUT }}$ & $\mathrm{U}(1)_{5}$ \\
\hline$H$ & $\mathbf{5}$ & $\mathbf{1}$ & 2 \\
$\Sigma$ & $\mathbf{2 4}$ & $\mathbf{1}$ & 0 \\
\hline$H^{\prime}$ & $\mathbf{1}$ & $\mathbf{4}$ & $-5 / 2$ \\
$\Xi^{\prime}$ & $\mathbf{1}$ & $\mathbf{1 5}$ & 0 \\
\hline
\end{tabular}

Table 2. Charge assignment of fermions and scalars in the minimal SU $(5)_{\text {GUT }} \times \mathrm{SU}(4)_{\text {DGUT }}$ unified model. The upper rows of the tables show the assignment in the $\mathrm{SU}(5)_{\mathrm{GUT}}$ sector while the lower rows show that in the $\mathrm{SU}(4)_{\mathrm{DGUT}}$ sector.

Let us consider the generic Lagrangian density that is invariant under $\mathrm{SU}(5)_{\mathrm{GUT}} \times$ $\mathrm{SU}(4)_{\mathrm{DGUT}}$. We also assume that the global "fiveness" $\mathrm{U}(1)_{5}$ is softly broken by Majorana masses $M_{R}$ for $\bar{N}{ }^{4}$ Yukawa interactions for dark fermions are given by

$$
\mathcal{L}_{\text {Yukawa }}=-Y_{D} \epsilon^{\alpha \beta \gamma \delta} H_{\alpha}^{\prime} Q_{U[\beta \gamma]}^{\prime} Q_{D \delta}^{\prime}-Y_{\bar{D}} H^{\prime \dagger \alpha} Q_{U[\alpha \beta]}^{\prime} \bar{Q}_{\bar{D}}^{\prime}{ }^{\beta}-Y_{N} H_{\alpha}^{\prime} \bar{Q}_{\bar{D}}^{\prime} \alpha \bar{N}+\text { h.c. },
$$

where the Greek letters $\alpha, \beta, \cdots=1, \cdots, 4$ are $\mathrm{SU}(4)_{\mathrm{DGUT}}$ indices and $\epsilon^{\alpha \beta \gamma \delta}$ is the totally antisymmetric tensor of $\mathrm{SU}(4)_{\mathrm{DGUT}}$. A square bracket $[. .$.$] represents antisymmetric$ indices.

Below the energy scale of the mass of $\phi_{C}$, denoted by $M_{C}$, the relevant effective Lagrangian density for portal interactions is given by

$$
\mathcal{L}_{\text {portal }}=\frac{Y_{N} Y_{\bar{D}}}{\sqrt{2} M_{C}^{2}} \epsilon_{a b c}\left(\bar{U}^{\prime a} \bar{D}^{\prime b}\right)\left(\bar{D}^{\prime c} \bar{N}\right)-\frac{Y_{N} Y_{D}^{*}}{\sqrt{2} M_{C}^{2}} \epsilon_{a b c}\left(U^{\prime \dagger a} D^{\prime \dagger b}\right)\left(\bar{D}^{\prime c} \bar{N}\right)+\text { h.c. },
$$

or below the energy scale of $M_{R}$,

$$
\mathcal{L}_{\text {portal }}=-\frac{Y_{N} Y_{\bar{D}} y_{N}}{\sqrt{2} M_{C}^{2} M_{R}} \epsilon_{a b c}\left(\bar{U}^{\prime a} \bar{D}^{\prime b}\right) \bar{D}^{\prime c}(L H)+\frac{Y_{N} Y_{D}^{*} y_{N}}{\sqrt{2} M_{C}^{2} M_{R}} \epsilon_{a b c}\left(U^{\prime \dagger a} D^{\prime \dagger b}\right) \bar{D}^{\prime c}(L H)+\text { h.c. }
$$

Here, $a, b, c=1,2,3$ represent $\mathrm{SU}(3)_{D}$ indices and $\epsilon_{a b c}$ is the totally antisymmetric tensor of $\mathrm{SU}(3)_{D}$. These are exactly what transfers the $B-L$ asymmetry generated by thermal leptogenesis into the dark sector [see eqs. (2.5) and (2.6)]. Although the massive gauge

\footnotetext{
${ }^{4} \mathrm{U}(1)_{5}$ can be a gauge symmetry since the gauge anomalies are cancelled if one introduces three generations of the right-handed neutrinos. When we consider the gauged $\mathrm{U}(1)_{5}, M_{R}$ is generated from a VEV of a $\mathrm{U}(1)_{5}$ breaking scalar field.
} 
bosons associated with $\mathrm{SU}(4)_{\mathrm{DGUT}}$ breaking also give rise to four-Fermi operators, they are irrelevant for the portal interactions. There arise no harmful portal operators that could wash out the $B-L$ asymmetry in combination with eq. (3.6). The phenomenological constraint eq. (2.7) reads

$$
M_{R}<M_{C} \lesssim 10\left(Y_{N} Y_{D, \bar{D}}\right)^{1 / 2} M_{R}, \quad \text { and thus } \quad Y_{N} Y_{D, \bar{D}} \gtrsim 0.01 .
$$

We remark that $M_{C}$, which is expected to be of the order of $v_{15}=\mathcal{O}\left(10^{10}\right) \mathrm{GeV}$, is larger than $M_{R} \sim 10^{9} \mathrm{GeV}$ required for thermal leptogenesis.

Dark nucleons can decay via the massive gauge bosons from SU(4) DGUT $_{\text {when dark }}$ electron $E^{\prime}$ is lighter than the dark nucleons. Since the dark GUT scale is much lower than the visible GUT scale and the dark dynamical scale $\Lambda_{\mathrm{DQCD}}$ is 10 times larger than the QCD scale, the DM decays within the age of the Universe:

$$
\tau\left(n^{\prime} \rightarrow E^{\prime}+\pi^{\prime}\right) \sim \frac{M_{\mathrm{DGUT}}^{4}}{g_{U}^{\prime 4} \Lambda_{\mathrm{DQCD}}^{5}} \sim 5 \times 10^{7} \mathrm{yr}\left(\frac{M_{\mathrm{DGUT}}}{10^{10} \mathrm{GeV}}\right)^{4}\left(\frac{2 \mathrm{GeV}}{\Lambda_{\mathrm{DQCD}}}\right)^{5},
$$

where $g_{U}^{\prime}$ is the $\mathrm{SU}(4)_{\text {DGUT }}$ gauge coupling at the scale of $M_{\mathrm{DGUT}}$. The $\mathrm{SU}(3)_{D}$ finestructure constant $\alpha_{S}^{\prime-1}$ vanishes at the dark dynamical scale $\Lambda_{\mathrm{DQCD}} \sim 2 \mathrm{GeV}$, which determines the value of $\alpha_{S}^{\prime}$ at the dark GUT scale, i.e., $M_{\text {DGUT }}$. We obtain $g_{U}^{\prime 2} \sim 0.38$ assuming $M_{\text {DGUT }} \sim 10^{10} \mathrm{GeV}$ and $\Lambda_{\mathrm{DQCD}} \sim 2 \mathrm{GeV}$.

To avoid this problem, we thus assume that the dark electron obtains a heavy mass via $\mathrm{SU}(4)_{\mathrm{DGUT}}$ symmetry breaking for simplicity, that is, $m_{E}^{\prime} \gg m_{p^{\prime}, n^{\prime}}$. In this case, the tiny dark quark current mass in eq. (2.1) requires fine-tuning between the vector-like mass term $Q_{D}^{\prime} \bar{Q}_{\bar{D}}^{\prime}$ and the Yukawa coupling $Q_{D}^{\prime} \Xi^{\prime} \bar{Q}_{\bar{D}}^{\prime}$, to give the dark electron mass of the order of $v_{15}$.

\subsection{Dark matter phenomenology}

Last but not least, we discuss the decay of the heavier dark nucleon. The heavier dark nucleon decays into the lighter one by emitting the dark photon in this model when the mass difference between the dark nucleons is larger than the mass of the dark photon $m_{\gamma^{\prime}}$. This process is induced by the mixing between the dark pion and the $\mathrm{U}(1)_{D}$ breaking Higgs. In this paper, we consider the case that this decay channel is open for simplicity. ${ }^{5}$

Let us consider the interaction between the dark nucleons and the dark photon. At the leading order the dark pions couple to the axial dark nucleon current, and the interaction is given by

$$
\mathcal{L}_{\pi^{\prime} N^{\prime}}=-\frac{g_{A}}{f_{\pi^{\prime}}} D_{\mu} \pi^{\prime a}\left(N^{\prime \dagger} \bar{\sigma}^{\mu} \tau^{a} N^{\prime}-\bar{N}^{\prime} \sigma^{\mu} \tau^{a} \bar{N}^{\prime \dagger}\right)
$$

where $g_{A}$ and $f_{\pi^{\prime}}$ are an axial coupling constant of the dark nucleons and a dark pion decay constant, respectively. $D_{\mu}$ is the covariant derivative of the dark pions. $N^{\prime}$ and $\bar{N}^{\prime}$ are

\footnotetext{
${ }^{5}$ When the mass difference is smaller than $m_{\gamma^{\prime}}$, the heavier dark nucleon decays into the lighter one with a pair of electron and positron via the dark photon-visible photon mixing instead of decaying with emitting the dark photon [36].
} 
dark isospin doublets, and the dark pion multiplet $\pi^{\prime}$ is defined as

$$
\pi^{\prime a} \tau^{a}=\frac{1}{\sqrt{2}}\left(\begin{array}{cc}
\pi^{\prime 0} & \sqrt{2} \pi^{\prime+} \\
\sqrt{2} \pi^{\prime-} & -\pi^{\prime 0}
\end{array}\right)
$$

where $\tau^{a}$ are the generators of dark isospin $\mathrm{SU}(2)$. The superscripts indicate the $\mathrm{U}(1)_{D}$ charges.

When there are parity violating masses for the dark quarks, the $\mathrm{U}(1)_{D}$ charged darkpion $\pi^{\prime+}$ gets a VEV. The chiral Lagrangian density for the dark pions is given by

$$
\begin{aligned}
\mathcal{L}_{\chi \mathrm{PT}} & =\frac{f_{\pi^{\prime}}^{2}}{4} \operatorname{tr}\left(\partial_{\mu} U_{\pi^{\prime}} \partial^{\mu} U_{\pi^{\prime}}^{\dagger}\right)+\left[B \operatorname{tr}\left(M U_{\pi^{\prime}}\right)+\text { h.c. }\right]+\cdots, \\
U_{\pi^{\prime}} & =\exp \left(\frac{i \pi^{\prime a} \tau^{a}}{f_{\pi^{\prime}}}\right), \quad M=\left(\begin{array}{cc}
m_{U^{\prime}} & Y_{D} v_{D} \\
Y_{\bar{D}} v_{D} & m_{D^{\prime}}
\end{array}\right),
\end{aligned}
$$

with the dark quark current masses $m_{U^{\prime}}$ and $m_{D^{\prime}}$, and the $\mathrm{U}(1)_{D}$ breaking Higgs VEV $v_{D}$. $B$ is a dimensionful parameter of the order of $f_{\pi^{\prime}}^{3}$. Expanding $U_{\pi^{\prime}}$, we obtain the dark pion mass term,

$$
\mathcal{L}_{\pi^{2}}=i \Delta m_{\pi^{\prime}}^{* 2} v_{D} \pi^{\prime+}-i \Delta m_{\pi^{\prime}}^{2} v_{D} \pi^{\prime-}-\frac{1}{2} m_{\pi^{\prime}}^{2}\left(\pi^{\prime 0}\right)^{2}-m_{\pi^{\prime}}^{2} \pi^{\prime+} \pi^{\prime-},
$$

where

$$
\Delta m_{\pi^{\prime}}^{2} \equiv \frac{B}{f_{\pi^{\prime}}}\left(Y_{\bar{D}}^{*}-Y_{D}\right), \quad m_{\pi^{\prime}}^{2} \equiv \frac{B\left(m_{U^{\prime}}+m_{D^{\prime}}\right)}{2 f_{\pi^{\prime}}^{2}}
$$

The dark pion gets a VEV,

$$
\left\langle{\pi^{\prime}}^{+}\right\rangle \equiv i v_{\pi}=\frac{i \Delta m_{\pi^{\prime}}^{2}}{m_{\pi^{\prime}}^{2}} v_{D}
$$

Here, we implicitly assume that the $\mathrm{U}(1)_{D}$ breaking masses $Y_{D, \bar{D}} v_{D}$ are subdominant when compared to the current masses. Otherwise, the typical scale of the dark Higgs and the dark pions is expected to be of the order of the dark QCD scale since $\Delta m_{\pi^{\prime}}^{2} \sim \mathcal{O}\left(f_{\pi^{\prime}}^{2}\right)$ dominates their masses. In our scenario, the dark QCD scale is around $2 \mathrm{GeV}$, and thus the chiral symmetry is expected to be a good symmetry if the dark quark current masses are below $200 \mathrm{MeV}$. When we assume that the mass of the dark photon is of the order of $100 \mathrm{MeV}, Y_{D, \bar{D}} v_{D}$ is approximately given by

$$
Y_{D, \bar{D}} v_{D} \sim 11 \mathrm{MeV}\left(\frac{Y_{D, \bar{D}}}{0.1}\right)\left(\frac{7 \times 10^{-2}}{\alpha^{\prime}}\right)^{1 / 2}\left(\frac{m_{\gamma^{\prime}}}{100 \mathrm{MeV}}\right) .
$$

Thus, without assuming very tiny couplings $Y_{D, \bar{D}}{ }^{6}$ we can achieve the $\mathrm{U}(1)_{D}$ breaking masses that are an order of magnitude smaller than the dark quark current masses.

\footnotetext{
${ }^{6}$ Indeed, we cannot make $Y_{D, \bar{D}}$ tiny so that the $B-L$ asymmetry is efficiently transferred after thermal leptogenesis [see eq. (3.7)].
} 
Here, we use the $\mathrm{U}(1)_{D}$ fine-structure constant $\alpha^{\prime}$ estimated in the GUT framework in the following way. $\mathrm{As} \mathrm{U}(1)_{D}$ and $\mathrm{SU}(3)_{D}$ are unified into $\mathrm{SU}(4)_{\mathrm{DGUT}}$ at the dark GUT scale, $\alpha^{\prime}$ and $\alpha_{S}^{\prime}$ are identified there. Therefore, the low-energy value of $\alpha^{\prime}$ is given by

$$
\alpha^{\prime-1}\left(\Lambda_{\mathrm{DQCD}}\right)=\frac{8}{3} \frac{\left(b^{\prime}-b_{s}^{\prime}\right)}{2 \pi} \ln \left(\frac{M_{\mathrm{DGUT}}}{\Lambda_{\mathrm{DQCD}}}\right)
$$

Here, $b^{\prime}=23 / 24$ and $b_{s}^{\prime}=-29 / 3$ are the one-loop $\beta$ function coefficients of $\mathrm{U}(1)_{D}$ and $\mathrm{SU}(3)_{D}$ gauge couplings, respectively. The prefactor of $8 / 3$ arises from the $\mathrm{SU}(4)_{\mathrm{DGUT}}$ normalization.

After the dark pion gets a VEV, the dark photon gets its mass not only via the dark Higgs but also via the dark pion. The Nambu-Goldstone boson eaten by the dark photon is a mixture of the phase degrees of freedoms of them, and therefore the interaction in eq. (3.9) leads to the interaction between the dark photon and the dark nucleons,

$$
\mathcal{L}_{\gamma^{\prime} p^{\prime} n^{\prime}}=-\frac{g_{A} g_{D} v_{\pi^{\prime}}}{f_{\pi^{\prime}}} A_{\mu}^{\prime}\left(-p^{\prime \dagger} \bar{\sigma}^{\mu} n^{\prime}+\bar{p}^{\prime} \sigma^{\mu} \bar{n}^{\prime \dagger}\right)+\text { h.c. }
$$

This interaction leads to the prompt decay of the heavier dark nucleon to the lightest one in this model, when the mass difference between the dark nucleons is larger than $m_{\gamma^{\prime}}$.

The dark proton can interact with the SM proton via the kinetic mixing, and hence a constraint from the DM direct detection experiment is much stronger than other constraints unless DM consists predominantly of the dark neutrons [36]. If the masses of the dark quarks are dominated by $v_{D}$, the dark neutron and the dark proton significantly mix in the mass basis. In this case, a constraint from the DM direct detection experiment is stringent irrespectively of the DM constituent.

\subsection{Remarks}

Several comments are in order. As we mentioned in subsection 3.2, we assume that the dark electron gets a mass comparable to the dark GUT scale in order to ensure the longevity of ADM. The decay of dark nucleons in eq. (3.8) is kinematically prohibitted if the dark electron is heavier than the dark nucleons. ${ }^{7}$ In such a case, the dark electron can be much lighter than the right-handed neutrinos, and then the right-handed neutrinos can decay into the dark electron and the dark Higgs boson. Such decays of the right-handed neutrinos can generate the $B-L$ asymmetry in addition to their decays into the visible lepton and

\footnotetext{
${ }^{7}$ For example, we may extend the dark GUT model so that $\Xi^{\prime}$ is complex scalar in the adjoint representation of SU(4) DGUT with additional vector-like fermions $\left(X^{\prime}, \bar{X}^{\prime}\right)$ in the $(\mathbf{4}, \overline{\mathbf{4}})$ representations with a mass $M_{X^{\prime}}$. When we impose a chiral symmetry with the charge assignment of $\Xi^{\prime}(+1), Q_{D}^{\prime}(-1), \bar{Q}_{\bar{D}}^{\prime}(-1)$ and $Q_{U}^{\prime}(+1)$, the Yukawa couplings of the dark quarks to $\Xi^{\prime}$ s s are restricted to $y_{D}^{\prime} Q_{D}^{\prime} \Xi^{\prime} \bar{X}+y_{\bar{D}}^{\prime} X^{\prime} \Xi^{\prime} \bar{Q}_{\bar{D}}^{\prime}+$ h.c. The chiral symmetry may be softly broken by the mass of $Q_{U}^{\prime}$ and is spontaneously broken by the VEV of $\Xi^{\prime}$. The phenomenology of the Nambu-Goldstone mode will be close to that of QCD axion [96-99]. Then, the masses of $D^{\prime}$ and $E^{\prime}$ are given by $y_{D}^{\prime} y_{\bar{D}}^{\prime} v_{15}^{2} / M_{X^{\prime}}$ and $y_{D}^{\prime} y_{\bar{D}}^{\prime} v_{15}^{2} / M_{X^{\prime}}$, respectively, for $M_{X^{\prime}} \gg v_{15}$. It allows us to have the dark electron mass about an order of magnitude larger than the dark quark masses. When we arrange the dark quark masses in a sub-GeV to a few $\mathrm{GeV}$ range, the dark electron mass can be heavier than the dark nucleon masses, with which the dark nucleon decay is prohibited. Although we need to extend the charge assignment to allow $H^{\prime} \bar{Q}_{\bar{D}}^{\prime} \bar{N}$, we do not go into further details.
} 
the SM Higgs. ${ }^{8}$ A new portal operator also arises below the energy scale of $M_{R}$,

$$
\mathcal{L}_{\text {new portal }}=\frac{y_{N} Y_{N}}{M_{R}}\left(\phi_{D} E^{\prime}\right)(L H)+\text { h.c. }
$$

This cosmology is intensively studied in ref. [83]. This operator causes the dark electron decay into the SM neutrino with emitting the dark photon.

It might be tempting to consider the dark GUT model based on $\mathrm{SU}(5)_{\mathrm{DGUT}}$, where the dark quarks in table 1 are unified into the $\mathbf{1 0}$ and the $\overline{\mathbf{5}}$ representations of SU(5) DGUT, instead of SU(4) DGUT. However, it is difficult to make such a mirror model in the dark sector cosmologically viable. The dark neutrinos in SU(5) DGUT are massless up to lepton number violating operators as in the SM, and then they affect the effective number of neutrino species $N_{\text {eff }}$ and can behave like a hot component of DM as the SM neutrinos. Furthermore, the dark nucleon can decay into the dark neutrino and the dark pion within the age of the Universe via dark nucleon decay operators when the SU(5) DGUT GUT scale is close to $10^{10} \mathrm{GeV}$. The latter problem cannot be avoided since the dark neutrino cannot be made heavier than the dark nucleons unlike the dark electron.

\section{Supersymmetric realization}

Intermediate-scale SUSY is theoretically and phenomenologically well-motivated UV physics (see refs. [84-87] for reviews). It would be natural to consider a SUSY extension of our $\mathrm{SU}(5)_{\mathrm{GUT}} \times \mathrm{SU}(4)_{\mathrm{DGUT}}$ model. Indeed, gauge couplings in the visible sector are precisely unified into one at the GUT scale when SUSY is assumed. The non-renormalization theorem ensures our choices of the model parameters, such as fine-tuning for the GUT-scale splittings between $\phi_{C}$ and $\phi_{D}$ and between $D^{\prime}$ and $E^{\prime}$, against quantum corrections. Scalar interactions are also restricted due to SUSY, and therefore some of Higgs multiplets are naturally light up to the little hierarchy between the SUSY breaking scale and the electroweak or the dark QED breaking scales. Therefore, in this section, we consider a minimal SUSY realization of the composite ADM model.

We assume the minimal supersymmetric standard model (MSSM) in the visible sector, and a minimal SUSY extension of the dark sector. $Q_{U}^{\prime}, Q_{D}^{\prime}$, and $Q_{\bar{D}}^{\prime}$ are chiral superfields denoted by the same symbols as their fermionic components. It should be noted that we introduce more than one generations of vector-like $Q_{D}^{\prime}$ and $Q_{\bar{D}}^{\prime}$ so that the supersymmetric neutron chiral multiplets, i.e., $\epsilon^{a b c} U_{a}^{\prime} D_{b}^{\prime} D_{c}^{\prime}$ and $\epsilon_{a b c} \bar{U}^{\prime a} \bar{D}^{\prime b} \bar{D}^{c}$, are available. We take two generations in the following, although we suppress generation indices for the sake of notational simplicity. We introduce two fundamental dark Higgs superfields, $H^{\prime}$ and $\bar{H}^{\prime}$, and a dark adjoint superfield $\Xi^{\prime}$. One can refer to table 2 for the charge assignment again. The superpotential in the dark sector is given by

$$
\begin{aligned}
W= & \bar{H}^{\prime}\left(\mu+\lambda \Xi^{\prime}\right) H^{\prime}+W_{\Xi^{\prime}} \\
& +Y_{D} \epsilon^{\alpha \beta \gamma \delta} H_{\alpha}^{\prime} Q_{U[\beta \gamma]}^{\prime} Q_{D \delta}^{\prime}+Y_{\bar{D}} \bar{H}^{\prime \alpha} Q_{U[\alpha \beta]}^{\prime} \bar{Q}_{\bar{D}}^{\prime}{ }^{\beta}+Y_{N} H_{\alpha}^{\prime} \bar{Q}_{\bar{D}}^{\prime} \alpha \bar{N}
\end{aligned}
$$

\footnotetext{
${ }^{8}$ Since we introduce at least two generations for the right-handed neutrinos, the relative phase of $Y_{N}$ 's (again note that generation indices are suppressed) provides a $C P$ violation in the dark sector.
} 
Here, $W_{\Xi^{\prime}}$ denotes the superpotential including only $\Xi^{\prime}$, and we assume that the superpotential is invariant under the $\mathrm{SU}(5)_{\mathrm{GUT}} \times \mathrm{SU}(4)_{\mathrm{DGUT}}$ and global $\mathrm{U}(1)_{5}$ symmetries. The $Q_{U}^{\prime}, Q_{D}^{\prime}$, and $Q_{\bar{D}}^{\prime}$ have mass terms although they are not shown here. As in the previous section, the masses of $\phi_{C}$ and $\phi_{D}$ should be split. The mass splitting between $\phi_{C}$ and $\phi_{D}$ is realized when fine-tuning of $\mu=3 \lambda v_{15}$ is assumed. ${ }^{9}$

\subsection{Tiny kinetic mixing and $B-L$ portal operator}

The kinetic mixing in the SUSY model arises from

$$
\mathcal{L}_{\epsilon}=\int d^{2} \theta \frac{1}{M_{\mathrm{Pl}}^{2}} \operatorname{tr}\left(\mathcal{W}_{G} \Sigma\right) \operatorname{Tr}\left(\mathcal{W}_{D} \Xi^{\prime}\right)+\text { h.c. } \sim \int d^{2} \theta \frac{\epsilon}{4 \cos \theta_{W}} \mathcal{W} \mathcal{W}^{\prime}+\text { h.c. }
$$

where $\mathcal{W}$ and $\mathcal{W}^{\prime}$ are field strength chiral superfields of $\mathrm{U}(1)_{Y}$ and $\mathrm{U}(1)_{D}$, respectively. The mixing parameter $\epsilon$ is defined in eq. (3.2).

Below the energy scales of the masses of the dark colored Higgs triplet and the righthanded neutrinos, the following effective superpotential arises:

$$
W_{\text {eff. }}=-\frac{Y_{N} y_{N} Y_{\bar{D}}}{M_{C} M_{R}} \epsilon_{a b c} \bar{U}^{\prime a} \bar{D}^{\prime b} \bar{D}^{\prime c}\left(L H_{u}\right)
$$

where $H_{u}$ is one of the MSSM Higgs doublets. Again it should be noted that we introduce two generations of $D^{\prime}$ and $\bar{D}^{\prime}$ so that the portal interaction does not vanish. As in the case of a non-SUSY model, we simply assume that the dark electron chiral multiplet gets a mass of the order of the dark GUT scale in order to stabilize the dark neutron, and thus we omit the term like eq. (3.18).

Due to superpartners of dark fermions, the portal interaction arises at dimension six rather than dimension seven. This relaxes the phenomenological constraints eqs. (2.7) and (3.7) as

$$
M_{R}<M_{C} \lesssim 10^{2} Y_{N} Y_{D, \bar{D}} M_{R}, \text { and thus } Y_{N} Y_{D, \bar{D}} \gtrsim 0.01
$$

where we assume that $\tan \beta$, which is the ratio of the VEVs of the two Higgs doublets in the MSSM, is of the order of unity.

\subsection{Lightest supersymmetric particles in two sectros}

In SUSY extensions, the lightest supersymmetric particles (LSPs) would also be stable due to the R-parity (i.e., a discrete subgroup of $\left.\mathrm{U}(1)_{5}\right)$. In particular, we have two species of the LSPs both in the MSSM and in the dark sector. They could lead to the overclosure

\footnotetext{
${ }^{9}$ While we simply assume fine-tuned parameters in this section, we may naturally solve the mass splitting by introducing 20-dimensional chiral multiplets $\eta^{\prime}(\mathbf{2 0})$ and $\bar{\eta}^{\prime}(\overline{\mathbf{2 0}})$ and imposing a chiral symmetry of $\eta^{\prime}(+1)$, $\bar{\eta}^{\prime}(+1), H^{\prime}(-1), \bar{H}^{\prime}(-1)$, and $Q_{U}^{\prime}(+1)$. The chiral symmetry may be softly broken by the mass of $Q_{U}^{\prime}$. The $\overline{\mathbf{2 0}}$ representation in $\mathrm{SU}(4)_{\mathrm{DGUT}}$ does not have $\mathrm{SU}(3)_{D}$ singlets as a component, $\overline{\mathbf{2 0}} \rightarrow \overline{\mathbf{3}}_{1 / 3}+\mathbf{3}_{5 / 3}+\mathbf{6}_{1 / 3}+\mathbf{8}_{1}$. Therefore, the product of $\overline{\mathbf{2 0}} \times \mathbf{4}=\mathbf{1 5}+\mathbf{2 0 ^ { \prime }}+\mathbf{4 5}$ indicates that the superpotential Yukawa interactions of $W \supset \lambda^{\prime} H^{\prime} \bar{\eta}^{\prime} \Xi^{\prime}+\bar{\lambda}^{\prime} \bar{H}^{\prime} \eta^{\prime} \Xi^{\prime}$ give a mass only for $\phi_{C}$ after the $\mathrm{SU}(4)_{\text {DGUT }}$ breaking. This mechanism is similar to the missing-partner mechanism in $\mathrm{SU}(5)_{\text {GUT }}$ models $[88,89]$. Although we need to extend the charge assignment to allow $H^{\prime} \bar{Q}_{\bar{D}}^{\prime} \bar{N}$, we do not go into further details.
} 
of the Universe. Even if their fractions to the total DM abundance are subdominant, the late-time (over one second) decay of the heavier LSP into the lighter one can cause cosmological problems. The ratios are severely constrained by the big-bang nucleosynthesis and the spectral distortion of the cosmic microwave background, especially when their decay products are electromagnetically charged (see, e.g., refs. [90, 91]).

In our setup, such harmful late-time decays could take place since the MSSM and the dark sectors feebly interact with each other below the energy scale of the order of $M_{R}$. Indeed, there is no renormalizable interaction term between the visible and the dark sectors below it, if one turns off the kinetic mixing between the $\mathrm{U}(1)_{Y}$ and the $\mathrm{U}(1)_{D}$ vector multiplets. For instance, one may think that the LSPs would be harmless if we make the dark squark the LSP in the dark sector and reduce its relic abundance through its efficient annihilation. However, its lifetime induced by the operator eq. (4.3) is too long:

$$
\tau\left(\widetilde{Q}^{\prime} \rightarrow Q^{\prime} Q^{\prime} \widetilde{L} H_{u}\right) \sim \frac{2048 \pi^{5} \Lambda^{4}}{m_{\widetilde{Q}^{\prime}}^{5}} \sim 4 \times 10^{6} \sec \left(\frac{\Lambda}{10^{10} \mathrm{GeV}}\right)^{4}\left(\frac{m_{\widetilde{Q^{\prime}}}}{1 \mathrm{TeV}}\right)^{-5}
$$

where $m_{\widetilde{Q}}$ is the dark squark mass, and $\Lambda^{2}=M_{C} M_{R} / Y_{N} y_{N} Y_{\bar{D}}$.

Thus the kinetic mixing between the $\mathrm{U}(1)_{Y}$ and the $\mathrm{U}(1)_{D}$ vector multiplets plays two important roles in making our cosmological scenario viable. Its bosonic part, i.e., the kinetic mixing between the dark and the visible photons, transfers the dark sector entropy into the visible sector. Its fermionic part, i.e., the kinetic mixing between the bino and the dark photino, helps the heavier LSP decay into the lighter one with a sufficiently short lifetime.

If the pure bino and the dark photino are the LSPs in the MSSM and the dark sectors, respectively, their relic abundance tends to be overabundant. We, therefore, consider that the MSSM higgsinos are the LSP in the MSSM sector ${ }^{10}$ while the dark higgsino, which is the fermionic partner of $\mathrm{U}(1)_{D}$ breaking Higgs $\phi_{D}$, is the LSP in the dark sector. More specifically, we take a split spectrum of sparticles [92-95] for simplicity: the gauginos and the higgsino have masses of $\mathcal{O}(1) \mathrm{TeV}$ while all the scalar particles other than the SM Higgs and the $\mathrm{U}(1)_{D}$ breaking Higgs are much heavier than $\mathcal{O}\left(10^{2}\right) \mathrm{TeV}$. In this case, the dark higgsino decay into the MSSM higgsino via the bino-dark photino kinetic mixing: ${ }^{11}$

$$
\begin{aligned}
\tau\left(\widetilde{\phi}_{D} \rightarrow \phi_{D} H \widetilde{H}\right) & \sim \frac{8 \pi}{\epsilon^{2} \alpha_{Y} \alpha^{\prime} m_{\widetilde{\phi}_{D}}} \\
& \sim 2 \times 10^{-5} \sec \left(\frac{10^{-9}}{\epsilon}\right)^{2}\left(\frac{8 \times 10^{-2}}{\alpha^{\prime}}\right)\left(\frac{1 \mathrm{TeV}}{m_{\widetilde{\phi}_{D}}}\right),
\end{aligned}
$$

where $m_{\widetilde{\phi}_{D}}$ is the mass of the dark higgsinos, and $\alpha_{Y} \simeq 0.01$ is the fine-structure constant of $\mathrm{U}(1)_{Y}$. We obtain the low-energy value of $\mathrm{U}(1)_{D}$ fine-structure constant, i.e., $\alpha^{\prime} \sim 8 \times 10^{-2}$, by setting $b^{\prime}=2$ and $b_{s}^{\prime}=-7$ instead in eq. (3.16) in the $\operatorname{SUSY~SU}(3)_{D} \times \mathrm{U}(1)_{D}$ dynamics. As a result, we find that the lifetime of the dark LSP decay through the kinetic mixing

\footnotetext{
${ }^{10}$ The LSP in the MSSM sector can be the neutral wino instead.

${ }^{11}$ We assume that the dark higgsino is heavier than the MSSM higgsino, although the following discussion is intact up to trivial modifications even if the MSSM higgsino is heavier than the dark higgsino.
} 
between the bino and the dark photino is much shorter than one second even though the mixing parameter is tiny.

Lastly, we comment on the dark LSP decay in the case with a light dark electron. The dark higgsino $\widetilde{\phi}_{D}$ can decay into the dark electron and the MSSM slepton and the MSSM Higgs doublets through the SUSY version of eq. (3.18). This decay process occurs faster than the decay through the kinetic mixing if the sleptons have the mass of the order of $\mathrm{TeV}$.

\section{Conclusion}

Composite ADM is an intriguing framework that naturally explains why the observed DM mass density is close to the baryon mass density. On the other hand, the mechanism requires both high-energy and low-energy portals between the visible and the dark sectors. The former transfers the asymmetry generated in one sector to the other, while the latter releases the resultant entropy of the dark sector into the visible sector. We have constructed UV completions of a composite ADM model to clarify the origin of the two portals.

Our model is based on a $\mathrm{SU}(5)_{\text {GUT }} \times \mathrm{SU}(4)_{\text {DGUT }}$ gauge dynamics. We have chosen the minimal candidate, i.e., the $\mathrm{SU}(4)_{\text {DGUT }}$ gauge dynamics, for GUT in the dark sector. $\mathrm{SU}(4)_{\mathrm{DGUT}}$ is broken into $\mathrm{SU}(3)_{D} \times \mathrm{U}(1)_{D}$ at an intermediate scale. $\mathrm{SU}(3)_{D}$ provides dark hadrons, including dark nucleons as the ADM candidate. Meanwhile the decay of the $\mathrm{U}(1)_{D}$ dark photon releases the entropy of the dark sector into the visible sector. The minimal dark quark contents are incorporated into vector-like representations of SU(4) $)_{\mathrm{DGUT}}$, i.e., $\mathbf{4}+\overline{\mathbf{4}}+\mathbf{6}$.

We have introduced a fundamental scalar field, whose $\mathrm{SU}(3)_{D}$ singlet component develops a VEV to give a mass to the dark photon. The high-energy portal interaction is mediated by the $\mathrm{SU}(3)_{D}$ triplet component of the fundamental scalar and the heavy righthanded neutrinos. Thanks to the global U(1) 5 symmetry, which is a GUT compatible extension of the $B-L$ symmetry, we can prohibit dangerous operators that carry different $B-L$ charges.

The low-energy portal, i.e., the kinetic mixing between the dark photon and the visible photon, is forbidden at the renormalizable level since both the $\mathrm{U}(1)$ photons are parts of larger non-Abelian gauge bosons. Above the GUT scale, we have naïvely expected that all non-renormalizable operators are suppressed by the Planck mass. We have obtained the preferable kinetic mixing of $\epsilon \sim 10^{-9}$ when the dark GUT scale is set to be about $10^{10} \mathrm{GeV}$. The mass of the $\mathrm{SU}(3)_{D}$ triplet component of the fundamental scalar is of the order of this dark GUT scale and is compatible with thermal leptogenesis as a production mechanism of the $B-L$ asymmetry.

We have also considered the SUSY extension of the UV model since SUSY plays an important role in the gauge coupling unification in the visible sector and the stability of the GUT-scale mass splittings against quantum corrections. However, the LSPs in both the sectors are long-lived and therefore could cause cosmological problems through their relic abundance and the late-time decay of the heavier LSP to the lighter LSP. We have found that a SUSY version of the above kinetic mixing plays another important role here. It leads to the kinetic mixing between the bino and the dark photino, through which 
the heavier LSP can decay into the lighter one with the lifetime much shorter than one second. Especially when we consider a split spectrum of sparticles, i.e., light gauginos, light higgsinos, and heavy scalars, the LSPs are cosmologically harmless.

\section{Acknowledgments}

This work is supported in part by Grants-in-Aid for Scientific Research from the Ministry of Education, Culture, Sports, Science, and Technology (MEXT) KAKENHI, Japan, No. 15H05889, No. 16H03991, No. 17H02878, and No. 18H05542 (M.I.) and by the World Premier International Research Center Initiative (WPI), MEXT, Japan. The work of A.K. and T.K. is supported by IBS under the project code, IBS-R018-D1. A.K. would like to acknowledge the European Centre for Theoretical Studies in Nuclear Physics and Related Areas $\left(\mathrm{ECT}^{*}\right)$ for its hospitality during the completion of this work.

Open Access. This article is distributed under the terms of the Creative Commons Attribution License (CC-BY 4.0), which permits any use, distribution and reproduction in any medium, provided the original author(s) and source are credited.

\section{References}

[1] S.B. Gudnason, C. Kouvaris and F. Sannino, Dark Matter from new Technicolor Theories, Phys. Rev. D 74 (2006) 095008 [hep-ph/0608055] [INSPIRE].

[2] D.D. Dietrich and F. Sannino, Conformal window of $\mathrm{SU}(N)$ gauge theories with fermions in higher dimensional representations, Phys. Rev. D 75 (2007) 085018 [hep-ph/0611341] [INSPIRE].

[3] M.Y. Khlopov and C. Kouvaris, Strong Interactive Massive Particles from a Strong Coupled Theory, Phys. Rev. D 77 (2008) 065002 [arXiv:0710.2189] [InSPIRE].

[4] M.Y. Khlopov and C. Kouvaris, Composite dark matter from a model with composite Higgs boson, Phys. Rev. D 78 (2008) 065040 [arXiv:0806.1191] [INSPIRE].

[5] R. Foadi, M.T. Frandsen and F. Sannino, Technicolor Dark Matter, Phys. Rev. D 80 (2009) 037702 [arXiv:0812.3406] [INSPIRE].

[6] J. Mardon, Y. Nomura and J. Thaler, Cosmic Signals from the Hidden Sector, Phys. Rev. D 80 (2009) 035013 [arXiv: 0905.3749] [INSPIRE].

[7] G.D. Kribs, T.S. Roy, J. Terning and K.M. Zurek, Quirky Composite Dark Matter, Phys. Rev. D 81 (2010) 095001 [arXiv:0909.2034] [INSPIRE].

[8] R. Barbieri, S. Rychkov and R. Torre, Signals of composite electroweak-neutral Dark Matter: LHC/Direct Detection interplay, Phys. Lett. B 688 (2010) 212 [arXiv:1001.3149] [INSPIRE].

[9] M. Blennow, B. Dasgupta, E. Fernandez-Martinez and N. Rius, Aidnogenesis via Leptogenesis and Dark Sphalerons, JHEP 03 (2011) 014 [arXiv:1009.3159] [INSPIRE].

[10] R. Lewis, C. Pica and F. Sannino, Light Asymmetric Dark Matter on the Lattice: SU(2) Technicolor with Two Fundamental Flavors, Phys. Rev. D 85 (2012) 014504 [arXiv:1109.3513] [INSPIRE]. 
[11] Lattice Strong Dynamics (LSD) collaboration, Lattice calculation of composite dark matter form factors, Phys. Rev. D 88 (2013) 014502 [arXiv: 1301.1693] [INSPIRE].

[12] A. Hietanen, R. Lewis, C. Pica and F. Sannino, Composite Goldstone Dark Matter: Experimental Predictions from the Lattice, JHEP 12 (2014) 130 [arXiv:1308.4130] [INSPIRE].

[13] J.M. Cline, Z. Liu, G. Moore and W. Xue, Composite strongly interacting dark matter, Phys. Rev. D 90 (2014) 015023 [arXiv:1312.3325] [InSPIRE].

[14] Lattice Strong Dynamics (LSD) collaboration, Composite bosonic baryon dark matter on the lattice: $\mathrm{SU}(4)$ baryon spectrum and the effective Higgs interaction,

Phys. Rev. D 89 (2014) 094508 [arXiv: 1402.6656] [INSPIRE].

[15] A. Hietanen, R. Lewis, C. Pica and F. Sannino, Fundamental Composite Higgs Dynamics on the Lattice: SU(2) with Two Flavors, JHEP 07 (2014) 116 [arXiv:1404.2794] [INSPIRE].

[16] G. Krnjaic and K. Sigurdson, Big Bang Darkleosynthesis, Phys. Lett. B 751 (2015) 464 [arXiv: 1406.1171] [INSPIRE].

[17] W. Detmold, M. McCullough and A. Pochinsky, Dark Nuclei I: Cosmology and Indirect Detection, Phys. Rev. D 90 (2014) 115013 [arXiv:1406.2276] [INSPIRE].

[18] W. Detmold, M. McCullough and A. Pochinsky, Dark nuclei II: Nuclear spectroscopy in two-color QCD, Phys. Rev. D 90 (2014) 114506 [arXiv:1406.4116] [INSPIRE].

[19] M. Asano and R. Kitano, Partially Composite Dark Matter, JHEP 09 (2014) 171 [arXiv: 1406.6374] [INSPIRE].

[20] J. Brod, J. Drobnak, A.L. Kagan, E. Stamou and J. Zupan, Stealth QCD-like strong interactions and the t $\bar{t}$ asymmetry, Phys. Rev. D 91 (2015) 095009 [arXiv:1407.8188] [INSPIRE].

[21] O. Antipin, M. Redi and A. Strumia, Dynamical generation of the weak and Dark Matter scales from strong interactions, JHEP 01 (2015) 157 [arXiv:1410.1817] [INSPIRE].

[22] E. Hardy, R. Lasenby, J. March-Russell and S.M. West, Big Bang Synthesis of Nuclear Dark Matter, JHEP 06 (2015) 011 [arXiv: 1411.3739] [INSPIRE].

[23] T. Appelquist et al., Stealth Dark Matter: Dark scalar baryons through the Higgs portal, Phys. Rev. D 92 (2015) 075030 [arXiv: 1503. 04203] [INSPIRE].

[24] T. Appelquist et al., Detecting Stealth Dark Matter Directly through Electromagnetic Polarizability, Phys. Rev. Lett. 115 (2015) 171803 [arXiv: 1503.04205] [INSPIRE].

[25] O. Antipin, M. Redi, A. Strumia and E. Vigiani, Accidental Composite Dark Matter, JHEP 07 (2015) 039 [arXiv: 1503.08749] [INSPIRE].

[26] E. Hardy, R. Lasenby, J. March-Russell and S.M. West, Signatures of Large Composite Dark Matter States, JHEP 07 (2015) 133 [arXiv: 1504.05419] [INSPIRE].

[27] R.T. Co, K. Harigaya and Y. Nomura, Chiral Dark Sector, Phys. Rev. Lett. 118 (2017) 101801 [arXiv: 1610. 03848] [INSPIRE].

[28] K.R. Dienes, F. Huang, S. Su and B. Thomas, Dynamical Dark Matter from Strongly-Coupled Dark Sectors, Phys. Rev. D 95 (2017) 043526 [arXiv:1610.04112] [INSPIRE].

[29] H. Ishida, S. Matsuzaki and Y. Yamaguchi, Bosonic-Seesaw Portal Dark Matter, Prog. Theor. Exp. Phys. 2017 (2017) 103B01 [arXiv: 1610.07137] [InSPIRE]. 
[30] S.J. Lonsdale, M. Schroor and R.R. Volkas, Asymmetric Dark Matter and the hadronic spectra of hidden QCD, Phys. Rev. D 96 (2017) 055027 [arXiv: 1704.05213] [InSPIRE].

[31] J.M. Berryman, A. de Gouvêa, K.J. Kelly and Y. Zhang, Dark Matter and Neutrino Mass from the Smallest Non-Abelian Chiral Dark Sector, Phys. Rev. D 96 (2017) 075010 [arXiv: 1706. 02722] [INSPIRE].

[32] M.I. Gresham, H.K. Lou and K.M. Zurek, Nuclear Structure of Bound States of Asymmetric Dark Matter, Phys. Rev. D 96 (2017) 096012 [arXiv: 1707. 02313] [INSPIRE].

[33] M.I. Gresham, H.K. Lou and K.M. Zurek, Early Universe synthesis of asymmetric dark matter nuggets, Phys. Rev. D 97 (2018) 036003 [arXiv: 1707.02316] [INSPIRE].

[34] A. Mitridate, M. Redi, J. Smirnov and A. Strumia, Dark Matter as a weakly coupled Dark Baryon, JHEP 10 (2017) 210 [arXiv:1707.05380] [INSPIRE].

[35] M.I. Gresham, H.K. Lou and K.M. Zurek, Astrophysical Signatures of Asymmetric Dark Matter Bound States, Phys. Rev. D 98 (2018) 096001 [arXiv:1805.04512] [InSPIRE].

[36] M. Ibe, A. Kamada, S. Kobayashi and W. Nakano, Composite Asymmetric Dark Matter with a Dark Photon Portal, JHEP 11 (2018) 203 [arXiv:1805.06876] [INSPIRE].

[37] E. Braaten, D. Kang and R. Laha, Production of dark-matter bound states in the early universe by three-body recombination, JHEP 11 (2018) 084 [arXiv:1806.00609] [INSPIRE].

[38] Y. Bai, A.J. Long and S. Lu, Dark Quark Nuggets, arXiv:1810.04360 [InSPIRE].

[39] A. Francis, R.J. Hudspith, R. Lewis and S. Tulin, Dark Matter from Strong Dynamics: The Minimal Theory of Dark Baryons, JHEP 12 (2018) 118 [arXiv:1809.09117] [INSPIRE].

[40] G.D. Kribs and E.T. Neil, Review of strongly-coupled composite dark matter models and lattice simulations, Int. J. Mod. Phys. A 31 (2016) 1643004 [arXiv:1604.04627] [INSPIRE].

[41] S. Nussinov, Technocosmology - could a technibaryon excess provide a "natural" missing mass candidate?, Phys. Lett. B 165 (1985) 55 [INSPIRE].

[42] S.M. Barr, R.S. Chivukula and E. Farhi, Electroweak Fermion Number Violation and the Production of Stable Particles in the Early Universe, Phys. Lett. B 241 (1990) 387 [INSPIRE].

[43] S.M. Barr, Baryogenesis, sphalerons, and the cogeneration of dark matter, Phys. Rev. D 44 (1991) 3062 [INSPIRE].

[44] D.B. Kaplan, Single explanation for both the baryon and dark matter densities, Phys. Rev. Lett. 68 (1992) 741 [INSPIRE].

[45] S. Dodelson, B.R. Greene and L.M. Widrow, Baryogenesis, dark matter and the width of the Z, Nucl. Phys. B 372 (1992) 467 [inSPIRE].

[46] V.A. Kuzmin, A Simultaneous solution to baryogenesis and dark matter problems, Phys. Part. Nucl. 29 (1998) 257 [hep-ph/9701269] [INSPIRE].

[47] M. Fujii and T. Yanagida, A Solution to the coincidence puzzle of $\Omega_{\mathrm{B}}$ and $\Omega_{\mathrm{DM}}$, Phys. Lett. B 542 (2002) 80 [hep-ph/0206066] [INSPIRE].

[48] R. Kitano and I. Low, Dark matter from baryon asymmetry, Phys. Rev. D 71 (2005) 023510 [hep-ph/0411133] [INSPIRE].

[49] G.R. Farrar and G. Zaharijas, Dark matter and the baryon asymmetry of the Universe, Phys. Rev. Lett. 96 (2006) 041302 [hep-ph/0510079] [INSPIRE]. 
[50] S.B. Gudnason, C. Kouvaris and F. Sannino, Towards working technicolor: Effective theories and dark matter, Phys. Rev. D 73 (2006) 115003 [hep-ph/0603014] [INSPIRE].

[51] R. Kitano, H. Murayama and M. Ratz, Unified origin of baryons and dark matter, Phys. Lett. B 669 (2008) 145 [arXiv:0807.4313] [InSPIRE].

[52] D.E. Kaplan, M.A. Luty and K.M. Zurek, Asymmetric Dark Matter, Phys. Rev. D 79 (2009) 115016 [arXiv:0901.4117] [InSPIRE].

[53] H. Davoudiasl and R.N. Mohapatra, On Relating the Genesis of Cosmic Baryons and Dark Matter, New J. Phys. 14 (2012) 095011 [arXiv:1203.1247] [INSPIRE].

[54] K. Petraki and R.R. Volkas, Review of asymmetric dark matter, Int. J. Mod. Phys. A 28 (2013) 1330028 [arXiv:1305.4939] [INSPIRE].

[55] K.M. Zurek, Asymmetric Dark Matter: Theories, Signatures and Constraints, Phys. Rept. 537 (2014) 91 [arXiv:1308.0338] [INSPIRE].

[56] S. Tulin and H.-B. Yu, Dark Matter Self-interactions and Small Scale Structure, Phys. Rept. 730 (2018) 1 [arXiv: 1705. 02358] [INSPIRE].

[57] M. Blennow, E. Fernandez-Martinez, O. Mena, J. Redondo and P. Serra, Asymmetric Dark Matter and Dark Radiation, JCAP 07 (2012) 022 [arXiv: 1203.5803] [INSPIRE].

[58] B. Holdom, Two U(1)'s and $\epsilon$ Charge Shifts, Phys. Lett. B 166 (1986) 196 [InSPIRE].

[59] D.S.M. Alves, S.R. Behbahani, P. Schuster and J.G. Wacker, Composite Inelastic Dark Matter, Phys. Lett. B 692 (2010) 323 [arXiv:0903.3945] [inSPIRE].

[60] D.S.M. Alves, S.R. Behbahani, P. Schuster and J.G. Wacker, The Cosmology of Composite Inelastic Dark Matter, JHEP 06 (2010) 113 [arXiv:1003.4729] [INSPIRE].

[61] M. Fukugita and T. Yanagida, Baryogenesis Without Grand Unification, Phys. Lett. B 174 (1986) 45 [INSPIRE].

[62] G.F. Giudice, A. Notari, M. Raidal, A. Riotto and A. Strumia, Towards a complete theory of thermal leptogenesis in the SM and MSSM, Nucl. Phys. B 685 (2004) 89 [hep-ph/0310123] [INSPIRE].

[63] W. Buchmüller, R.D. Peccei and T. Yanagida, Leptogenesis as the origin of matter, Ann. Rev. Nucl. Part. Sci. 55 (2005) 311 [hep-ph/0502169] [INSPIRE].

[64] S. Davidson, E. Nardi and Y. Nir, Leptogenesis, Phys. Rept. 466 (2008) 105 [arXiv: 0802.2962] [INSPIRE].

[65] M. Bauer, P. Foldenauer and J. Jaeckel, Hunting All the Hidden Photons, JHEP 07 (2018) 094 [arXiv: 1803.05466] [INSPIRE].

[66] J.H. Chang, R. Essig and S.D. McDermott, Revisiting Supernova 1987A Constraints on Dark Photons, JHEP 01 (2017) 107 [arXiv: 1611.03864] [INSPIRE].

[67] J.H. Chang, R. Essig and S.D. McDermott, Supernova 1987A Constraints on Sub-GeV Dark Sectors, Millicharged Particles, the QCD Axion and an Axion-like Particle, JHEP 09 (2018) 051 [arXiv: 1803.00993] [INSPIRE].

[68] P. Minkowski, $\mu \rightarrow$ e $\gamma$ at a Rate of One Out of $10^{9}$ Muon Decays?, Phys. Lett. B 67 (1977) 421 [INSPIRE].

[69] T. Yanagida, Horizontal gauge symmetry and masses of neutrinos, Conf. Proc. C $\mathbf{7 9 0 2 1 3 1}$ (1979) 95 [INSPIRE]. 
[70] M. Gell-Mann, P. Ramond and R. Slansky, Complex Spinors and Unified Theories, Conf. Proc. C 790927 (1979) 315 [arXiv: 1306.4669] [InSPIRE].

[71] S.L. Glashow, The Future of Elementary Particle Physics, NATO Sci. Ser. B 61 (1980) 687 [INSPIRE].

[72] M. Ibe, S. Matsumoto and T.T. Yanagida, The GeV-scale dark matter with $B-L$ asymmetry, Phys. Lett. B 708 (2012) 112 [arXiv:1110.5452] [INSPIRE].

[73] H. Fukuda, S. Matsumoto and S. Mukhopadhyay, Asymmetric dark matter in early Universe chemical equilibrium always leads to an antineutrino signal, Phys. Rev. D 92 (2015) 013008 [arXiv: 1411.4014] [INSPIRE].

[74] H. Georgi and S.L. Glashow, Unity of All Elementary Particle Forces, Phys. Rev. Lett. 32 (1974) 438 [INSPIRE].

[75] Super-Kamiokande collaboration, Search for proton decay via $p \rightarrow e^{+} \pi^{0}$ and $p \rightarrow \mu^{+} \pi^{0}$ in 0.31 megaton - years exposure of the Super-Kamiokande water Cherenkov detector, Phys. Rev. D 95 (2017) 012004 [arXiv: 1610.03597] [INSPIRE].

[76] H. Murayama and T. Yanagida, Viable SU(5) GUT with light leptoquark bosons, Mod. Phys. Lett. A 7 (1992) 147 [InSPIRE].

[77] I. Dorsner and P. Fileviez Pérez, Unification without supersymmetry: Neutrino mass, proton decay and light leptoquarks, Nucl. Phys. B 723 (2005) 53 [hep-ph/0504276] [INSPIRE].

[78] B. Bajc and G. Senjanović, Seesaw at LHC, JHEP 08 (2007) 014 [hep-ph/0612029] [INSPIRE].

[79] B. Bajc, M. Nemevšek and G. Senjanović, Probing the seesaw mechanism at CERN LHC, Phys. Rev. D 76 (2007) 055011 [hep-ph/0703080] [INSPIRE].

[80] M. Ibe, Small steps towards Grand Unification and the electron/positron excesses in cosmic-ray experiments, JHEP 08 (2009) 086 [arXiv:0906.4667] [INSPIRE].

[81] T. Aizawa, M. Ibe and K. Kaneta, Coupling Unification and Dark Matter in a Standard Model Extension with Adjoint Majorana Fermions, Phys. Rev. D 91 (2015) 075012 [arXiv: 1411.6044] [INSPIRE].

[82] P. Cox, A. Kusenko, O. Sumensari and T.T. Yanagida, SU(5) Unification with TeV-scale Leptoquarks, JHEP 03 (2017) 035 [arXiv: 1612.03923] [INSPIRE].

[83] A. Falkowski, J.T. Ruderman and T. Volansky, Asymmetric Dark Matter from Leptogenesis, JHEP 05 (2011) 106 [arXiv:1101.4936] [INSPIRE].

[84] H.P. Nilles, Supersymmetry, Supergravity and Particle Physics, Phys. Rept. 110 (1984) 1 [INSPIRE].

[85] H.E. Haber and G.L. Kane, The Search for Supersymmetry: Probing Physics Beyond the Standard Model, Phys. Rept. 117 (1985) 75 [InSPIRE].

[86] J. Wess and J. Bagger, Supersymmetry and supergravity, Princeton University Press, Princeton New Jersey U.S.A. (1992) [INSPIRE].

[87] S.P. Martin, A Supersymmetry primer, Adv. Ser. Direct. High Energy Phys. 21 (2010) 1 [Adv. Ser. Direct. High Energy Phys. 18 (1998) 1] [hep-ph/9709356] [INSPIRE].

[88] B. Grinstein, A Supersymmetric SU(5) Gauge Theory with No Gauge Hierarchy Problem, Nucl. Phys. B 206 (1982) 387 [INSPIRE]. 
[89] A. Masiero, D.V. Nanopoulos, K. Tamvakis and T. Yanagida, Naturally Massless Higgs Doublets in Supersymmetric SU(5), Phys. Lett. B 115 (1982) 380 [INSPIRE].

[90] V. Poulin, J. Lesgourgues and P.D. Serpico, Cosmological constraints on exotic injection of electromagnetic energy, JCAP 03 (2017) 043 [arXiv: 1610.10051] [INSPIRE].

[91] M. Kawasaki, K. Kohri, T. Moroi and Y. Takaesu, Revisiting Big-Bang Nucleosynthesis Constraints on Long-Lived Decaying Particles, Phys. Rev. D 97 (2018) 023502 [arXiv: 1709.01211] [INSPIRE].

[92] N. Arkani-Hamed and S. Dimopoulos, Supersymmetric unification without low energy supersymmetry and signatures for fine-tuning at the LHC, JHEP 06 (2005) 073 [hep-th/0405159] [INSPIRE].

[93] G.F. Giudice and A. Romanino, Split supersymmetry, Nucl. Phys. B 699 (2004) 65 [Erratum ibid. B 706 (2005) 487] [hep-ph/0406088] [INSPIRE].

[94] J.D. Wells, PeV-scale supersymmetry, Phys. Rev. D 71 (2005) 015013 [hep-ph/0411041] [INSPIRE].

[95] M. Ibe, T. Moroi and T.T. Yanagida, Possible Signals of Wino LSP at the Large Hadron Collider, Phys. Lett. B 644 (2007) 355 [hep-ph/0610277] [INSPIRE].

[96] R.D. Peccei and H.R. Quinn, CP Conservation in the Presence of Instantons, Phys. Rev. Lett. 38 (1977) 1440 [INSPIRE].

[97] R.D. Peccei and H.R. Quinn, Constraints Imposed by CP Conservation in the Presence of Instantons, Phys. Rev. D 16 (1977) 1791 [INSPIRE].

[98] S. Weinberg, A New Light Boson?, Phys. Rev. Lett. 40 (1978) 223 [inSPIRE].

[99] F. Wilczek, Problem of Strong $P$ and T Invariance in the Presence of Instantons, Phys. Rev. Lett. 40 (1978) 279 [InSPIRE]. 